\title{
DISTÚRBIO COGNITIVO TRANSITÓRIO ASSOCIADO A ATIVIDADE EPILEPTIFORME GENERALIZADA OU DIFUSA DURANTE O ELETRENCEFALOGRAMA
}

\author{
Lineu Corrêa Fonseca', Gloria M A S Tedrus', Diana Toselo Lalloni², \\ Lucinda Maria Garcia Tella³, Patricia Maluf, Valdemar Donizeti de Sousa ${ }^{4}$
}

\begin{abstract}
RESUMO - Atividade epileptiforme (AE) no eletrencefalograma (EEG) é achado comum entre as crises epilépticas e, menos freqüentemente, em outras condições clínicas. AE "subdínica" pode estar acompanhada de distúrbio cognitivo transitório (DCT). Foram estudados 30 pacientes ( 27 com epilepsia) com AE generalizada ou difusa, freqüente, em EEG inicial. Em EEG subseqüente, foi avaliado o desempenho em testes de classificação visual de animais e objetos, discriminação de cores, memória verbal e auditiva, reconhecimento de padrões e estimação de tempo. Foi comparado, estatisticamente, o número de respostas corretas ou incorretas durante a AE e fora dela. DCT foi caracterizado em 43,3\% dos casos. Em 23,3\% a AE foi rara, não permitindo avaliação de DCT. Assim, pôde ser caracterizado comprometimento transitório de atividades cognitivas durante AE até então considerada "subclínica". Há necessidade de pesquisar a importância desse fator em pacientes com comprometimento cognitivo e de caracterizar as situações de eventuais benefícios na medicação dirigida à $A E$.
\end{abstract}

PALAVRAS-CHAVE: epilepsia, eletrencefalograma, cognição, atividade epileptiforme

\section{Transient cognitive impairment during generalized or diffuse epileptiform EEG discharges}

\begin{abstract}
Epileptiform EEG discharges (EED) are a frequent finding in people with epilepsy, and may occur in other clinical conditions. Formal testing during $\mathbb{E G}$ reco rding may demonstrate transitory cognitive impairment (TCl). Thirty patients (27 with epilepsy) with generalized or diffuse EED on a previous examination w ere studied. The interaction between EED and cognitive tasks was evaluated on subsequent EEG. The numbers of correct or incorrect answers were compared with the occurrence or absence of EED. Results were analyzed by non parametric tests. TCI was characterized in $43.3 \%$ of the patients. In $23.3 \%$ the EED were rare and it was not possible to evaluate the occurrence of $\mathrm{TCl}$. The findings confirm that otherwise subclinical EED may be accompanied by $\mathrm{TCl}$. There is a need of researchs to evaluate the relevance of $\mathrm{TCl}$ and to determine when EED should be treated.
\end{abstract}

KEY WORDS: epilepsy, electroencephalography, epileptiform activity, cognition

Durante as crises epilépticas, o eletrencefalograma (EEG) registra alterações importantes que são expressão do processo fisiopatogênico básico da des ca rga neuronal anormal excessiva. O EEG também pode mostrar a "atividade epileptiforme" (AE) ent reas crises epilépticas. $O$ desenvolvimento de testes permitindo a avaliação de funções no curto perío do de duração da $A E$, no EEG intercrítico, evidenciou, em parte dos pacientes (30-50\% ), distúrbios em tempo de reação ${ }^{1,2}$, detecção de sinais ${ }^{3}$, testes de memória ${ }^{4}$, testes de atenção e sub-testes de inteligência 5 . Esse distúrbio cognitivo momentâneo que acompanha a $A E$, observado em testes, mas sem que haja crise epiléptica evidente, foi denominado distúrbio cognitivo transitório (DCT). É demonstrando com maior freqüência na $\mathrm{AE}$ generalizada e, principalmente, quando esta dura 3 ou mais segundos 6 .

Com a utilização de técnicas mais apuradas, foi também possível evidenciar DCT durante AE focal e constatar que pode haver especificidade do DCT segundo o hemisfério afetado pela $\mathrm{AE}^{7}$ : o hemisfério direito associado a comprometimento em tarefas envolvendo noções espaciais, e o hemisfério esquercb a tarefas verbais ${ }^{8,9}$. A importância prática do reconhecimento do DCT é reforçada pela com-

\footnotetext{
${ }^{1}$ P rofessor Titular de Neurologia da Faculdade de Medicina da Pontifícia Universidade Católica de Campinas, Campinas SP, Brasil (PUC-Campinas); ${ }^{2}$ P rofessor Titular de Psicologia (PUC-Campinas); ${ }^{3}$ P rofessor Assistente de Neurologia (PUC-Campinas); ${ }^{4}$ Bolsista de iniciação científica do CNPQ/DF - Conselho Nacional de Desenvolvimento Científico e Tecnológico/DF.
} 
p rovação do comprometimento de desempenho em situações do cotidiano como a leitura e direção de veículos ${ }^{9-12}$ e pelo fato de já ter sido demonstrado DCT em pacientes sem epilepsia declarada ${ }^{7}$. Os estudos sobre DCT podem contribuir para avaliar dificuldades de aprendizado/comportamento que são relativamente comuns em pacientes com epilepsia ${ }^{13}$. Importa assinalar que AE pode ser observada em porcentagem pequena (cerca de 3\%) de crianças "normais" ${ }^{14}$, mas de modo mais freqüente, mesmo na ausência de epilepsia, em crianças com distúrbio de aprendizado/comportamento ${ }^{15,16}$. Recentemente tem sido mostrada a redução da $A E$ com algumas drogas antiepilépticas ${ }^{17,18}$, o que tem trazido benefícios cognitivos aos pacientes, em certas condições clínicas ${ }^{18,19}$.

As pesquisas sobre DCT são ainda pouco numerosas e apresentam limitações em vários aspectos, como o número de indivíduos, distinção entre DCT e crise evidente e o tipo de casuística estuda$\mathrm{da}^{6,20,21}$,

O objetivo desta pesquisa é estudar a ocorrência de DCT associado a AE generalizada ou difusa com a aplicação de vários testes cognitivos.

\section{MÉTODO}

Foram estudados 30 pacientes examinados, em seqüência, no Serviço de Eletrencefalografia e ambulatórios/enfemarias de neurologia do Hospital e Maternidade Celso Pierro (PUC-Campinas) que apresentavam atividade epileptiforme (AE) freqüente, generalizada ou difusa, no EEG. Foram incluídos os pacientes que tinham condições de colaboração e compreensão compatíveis com as exigências das provas a serem realizadas durante o EEG.

Foram levantados os dados relativos à história, exame físico/neurológico, exames subsidiáriose diagnósticos etiológico e sindrômico.

Foi realizada anamnese sobre as crises epilépticas do paciente, seus antecedentes pessoais e familiares, além de situações de seu cotidiano que pudessem ser manifestações sutis decorrentes dos mecanismos fisiopatogênicos próprios da epilepsia.

O EEG de rotina compreendeu: repouso, hiperventilação, abertura e fechamento dos olhos e fotoestimulação intermitente.

Quanto à $A E$, foi considerada generalizada a que o co mia em todas as regiões da cabeça e difusa a que era registrada em áreas amplas de um ou de ambos os lados da cabeça22.

Os pacientes nos quais foi constatada AE generalizada ou difusa, freqüente no EEG de rotina, foram solicitados a realizar um EEG durante atividades cognitivas. Para este teste foi utilizado o sistema M indTracer (Neuronic SA) que envolveu dois mi orocomputadores interligados: um deles destinado à produção e apresentação de estímulos assim como reg istro das respostas e o outro para a captação, monitorização e armazenamento dos dados da atividade elétrica cerebral registrada em 21 canais (sistema 10-20). A cada estímulo produzido pelo primeiroequipamento correspondeu uma marca no traçado da atividade elétrica cerebral registrada pelo segundo. Conforme as respostas fossem corretas ou incorretas, correspondiam marcas diferentes no traçado.

Foram utilizados os seguintes testes

1. Estimação de tempo (ET) - Durante a prova aparece um pequeno retângulo que se move horizontalmente da esquerda para a direita, no monitor. No seu trajeto, o retângulo atravessa uma faixa escura na qual ele não é visível, e o sujeito deve detectar quando o retângulo rea parecerá novamente pressionando uma tecla; se a resposta for correta, o retângulo reaparecerá com outra cor, do contrário, se emite um som. É prova elaborada para avaliar operações perceptuais elementares de organização espaço-temporal da informação.

2. Reconhecimento de padrões (RP) - O sujeito deve memorizar o primeiro padrão apresentado na tela. Após um som emitido, aparece o segundo padrão que deverá ser comparado com o primeiro; o sujeito terá que pressionar determinadas tedas segundo o estímulo seja ou não idêntico ao inicial. É uma tarefa de memória a curto prazo.

3. Discriminação de cores (DC) - São apresentadas imagens de aviões de diversas cores e o paciente deve usar uma determinada tecla para uma cor previamente selecionada e outra tecla para as demais cores.

4. Classificação visual animal/objeto (CVAO) - São apresentadas imagens de animais ou objetos e o paciente faz a discriminação entre essas duas categorias, acionando teclas previamente estipuladas.

5. Memória auditiva e visual (MAMV) - É emitido um sinal sonoro (apito) e após é liberada a seqüência de estímulos constituída por 3 palavras faladas e 3 imagens intercaladas. Após um intervalo de 5 segundos o sujeito ouvirá 8 palavras e verá 8 imagens; haverá somente palavra e uma imagem previamente apresentadas. Quando o paciente ouvir a palavra ou vir a imagem já apresentada, deverá apertar uma tecla estabelecida e em caso negativo, outra tecla.

6. Memória Visual (MV) e Memória Auditiva (MA) Nestes testes, após um sinal sonoro de aviso, é apresentada uma imagem (MV) ou, respectivamente, uma palavra falada (MA) e depois são apresentadas 5 imagens (MV) ou 5 palavras (MA), dentre as quais, aquela previamente mostrada. O sujeito deverá acionar uma de duas teclas, segundo o estímulo tenha sido previa mente apresentado ou não. 
Para a pesquisa de DCT é fundamental que a tarefa permita, pelo paciente, acerto na maioria dasrespostas a fim de permitir surpreender eventual desempenho estatisticamente inferior durante a $A E$ em relação ao período sem $A E^{7,9,23}$. Se a tarefa for muito difícil para o indivíduo, os erros já dominarão nos trechos sem $A E$ e não haverá como identificar eventual queda de produção durante a AE. Se a tarefa for muito fácil, levando a acerto de $100 \%$ das respostas, pode ser que a AE tenha menos possibilidade de interferir com o desempenho devido à grande facilidade do processamento da tarefa.

Levando em conta essas considerações, foram escolhidos tipo e características (duração e intervalo entre estímulos, proporção de repostas "corretas" e incorretas" esperadas, duração da prova) das tarefas que permitissem acerto na maioria das respostas. Para isso foram realizados testes breves até que fosse possível identificar a situação de teste que permitia ao individuo acertar a maioria das respostas; esta situação foi a utilizada durante a pesquisa. As tarefas foram, assim, individualizadas de acordo com fatores como idade, capacidades cognitivas e familiaridade com informática. Para as crianças, por exemplo, a prova mais utilizada, pela sua maior simplicidade foi a de classificação entre animais e objetos.

Durante os testes, um dos autores deste trabalho obsenava atentamente o paciente visando detectar sinais de crise epiléptica. Ao final do teste, solicitava-se ao paciente que referisse eventuais sintomas que pudessem ser atribuídos a crises.

$\mathrm{Na}$ análise dos dados, foram comparados os errose acertos entre os períodos livres de $A E$ e os períodos com $\mathrm{AE}$, com o teste do $\chi 2$ ou o teste de Fisher, para nível de significância de 0,05. Em acordo com a literatura sobre o tema $7,23,24$, foi considerado haver DCT, quando se constatou, de modo estatisticamente significativo, desempenho inferior na ocorrência de respostas corretas durante a $A E$ do que fora da $A E$.

Foi também levado em conta o momento em que a AE ocorreu - durante apresentação de estímulo, entre estímulo e resposta e durante a resposta.

Este esquema de caracterização de DCT, no qual o paciente necessariamente serve como seu próprio cont role, elimina diferentes fatores de confusão como idade, tipos de epilepsia ou medicação ${ }^{24}$.

O projeto de pesquisa foi aprovado pelo Comitê de Ética em Pesquisa da PUC-Campinas.

\section{RESULTADOS}

Foram estudados 30 pacientes (18 do sexo feminino e 12 do masculino) que apresentaram, em EEG anterior, AE freqüente generalizada ou difusa.

Nas tabelas 1 e 2 constam, respectivamente, em crianças/adolescentese adultos, a Idade, śndro me epiléptica, características da atividade epileptiforme, testes utilizado durante o EEG e o resultado do teste em termos de significância estatística. Os pacientes com epilepsia tinham prescrição de medicação anti-epiléptica (carbamazepina, fenobarbital, fenitoína, ácido valpróico) enquanto aqueles sem crise epilépticas não faziam uso da mesma.

Na Tabela 3 estão apresentados, de modo resumido, os resultados da pesquisa.

Tabela 1. Idade, síndrome epiléptica, características da atividade epileptiforme durante o teste, testes utilizados e resultados na pesquisa de distúrbio cognitivo transitório (DCT) em crianças e adolescentes.

\begin{tabular}{|c|c|c|c|c|c|c|}
\hline Caso & $\begin{array}{l}\text { Idade } \\
\text { (anos) }\end{array}$ & Síndrome epiléptica & $\begin{array}{c}\text { Atividade epileptiforme } \\
\text { Tipo e local }\end{array}$ & $\begin{array}{l}\text { e }(\mathrm{AE}) \\
\text { Duração }\end{array}$ & Teste & Resultados \\
\hline 1 & 15 & FS & $\mathrm{PO}, \mathrm{HD}$ & $1-5 s$ & CVAO* & Com DCT* \\
\hline 2 & 9 & FPS & $\mathrm{P}, \mathrm{HD}$ & $0,3-3 s s$ & CVAO & Sem DCT \\
\hline 4 & 16 & FS & OA, frontotemporal D & $0,5-2 \mathrm{~s}$ & CVAO/MV/MA & Sem DCT \\
\hline 9 & 9 & FS & PO, Dif & $1-3 \mathrm{~s}$ & DC/CVAO* & Com DCT* \\
\hline 13 & 11 & FS & P e PP, bilateral & $0,5-3 \mathrm{~s}$ & DC & Com DCT* \\
\hline 14 & 12 & $\mathrm{Gl}$ & PO 4-5Hz,PPO, Gen & & CVAO/DC/MA & AE rara \\
\hline 17 & 6 & Sem epilepsia & P, centrotemporal D & $0,3-2 \mathrm{~s}$ & CVAO & Sem DCT \\
\hline 18 & 13 & FPS & P, bilateral & $0,3-3 \mathrm{~s}$ & DC/CVAO* & Sem DCT \\
\hline 19 & 9 & $\mathrm{Gl}$ & PO 3Hz, Gen & & CVAO/RP/ET & AE rara \\
\hline 20 & 11 & FPS & PO, bilateral, Dif & $1-7 \mathrm{~s}$ & CVAO* & Com DCT* \\
\hline 21 & 12 & $\mathrm{Gl}$ & PO, Gen & $2-8 s$ & RP/ET* & Com DCT* \\
\hline 24 & 13 & FPS & P, bilateral & $0,4-3 \mathrm{~s}$ & CVAO/DC & sem DCT \\
\hline 28 & 12 & $\mathrm{GI}$ & PO, Dif & 3-8 s & CVAO/ET/RP* & Com DCT* \\
\hline 29 & 11 & Sem epilepsia & PO, Dif & $1-8 \mathrm{~s}$ & ET/DC/CVAO* & Com DCT* \\
\hline 30 & 18 & Gl, ausência & PO, Dif & & CVAO/MA & Com crise \\
\hline
\end{tabular}

FS, focal sintomática; FPS, focal provavelmente sintomática; Gl, generalizada idiopática; GPS, generalizada provavelmente si ntomática; P, ponta; PO, complexo de ponta-onda; PP, poliponta; AO, onda aguda; Gen, generalizada; Dif, difusa; HD, hemisfério direito; $\mathrm{DCT}$, distúrbio cognitivo transitório; $\mathrm{AE}$, atividade epileptiforme; $\mathrm{CVAO}$, classificação visual de animais e objetos; ET, estimativa de tempo; DC discriminação de cores; RP, reconhecimento de padrão; MV, memória verbal; MA, memória auditiva.*menor proporção de respostas corretas durante a $A E$ do que fora da $A E$ (qui-quadrado ou Fisher, $p \varangle 0,05$ ). 
Tabela 2. Idade, síndrome epiléptica, características da atividade epileptiforme durante o teste, testes utilizados e resultado na pesquisa de distúrbio cognitivo transitório (DCT) em adultos.

\begin{tabular}{|c|c|c|c|c|c|c|}
\hline \multirow[t]{2}{*}{ Caso } & \multirow{2}{*}{$\begin{array}{l}\text { Idade } \\
\text { (anos) }\end{array}$} & \multirow[t]{2}{*}{ Síndrome epiléptica } & Atividade epileptiforme & \multirow{2}{*}{$\begin{array}{c}\text { (AE) } \\
\text { Duração }\end{array}$} & \multirow[t]{2}{*}{ Teste } & \multirow[t]{2}{*}{ Resultados } \\
\hline & & & Tipo e local & & & \\
\hline 3 & 50 & Gl, ausência & PO 5Hz, Gen & & CVAO/MV & AE rara \\
\hline 5 & 19 & GI & PO, Gen & $1 \mathrm{~s}$ & MVMA* & Com DCT* \\
\hline 6 & 46 & FS & OA-onda lenta, Dif & $0,5-3 \mathrm{~s}$ & CVAO/MV & Sem DCT \\
\hline 7 & 33 & GPS & PO e PPO, Gen & $0,5-5 \mathrm{~s}$ & CVAO/MA & Sem DCT \\
\hline 8 & 38 & FS & OA, temporais & $0,5-2 \mathrm{~s}$ & $\mathrm{MA} / \mathrm{MV}$ & AE rara \\
\hline 10 & 37 & FS & OA, frontotemporal, Dif & $1-2 \mathrm{~s}$ & $M V^{*}$ & Com DCT* \\
\hline 11 & 24 & FPS & PO, bilateral & & CVAO/DC/AD & AE rara \\
\hline 12 & 30 & $\mathrm{Gl}$ & PO, Gen & $5 \mathrm{~s}$ & CVAO* & Com DCT* \\
\hline 15 & 43 & Sem epilepsia & PO 5Hz, PPO, Dif & $8 \mathrm{~s}$ & CVAO/RP* & Com DCT* \\
\hline 16 & 36 & Gl, ausência & PPO irregular, Gen & & $\mathrm{RP} / \mathrm{ET}$ & AE rara \\
\hline 22 & 36 & FS & PO irregular, bilateral & $0,7-3 \mathrm{~s}$ & $\mathrm{ET} / \mathrm{RP}$ & Sem DCT \\
\hline 23 & 45 & GI & PO e PPO, Gen & $1-3 s$ & CVAO/DC* & Com DCT* \\
\hline 25 & 32 & FS & PO irregular, bilateral & $0,5-3 \mathrm{~s}$ & CVAO/ET/RP & Sem DCT \\
\hline 26 & 21 & FS & OA, bilateral & & CVAO/DC & AE rara \\
\hline 27 & 47 & FS & PO temporais & $1-2 \mathrm{~s}$ & CVAO/ET/DC* & Com DCT* \\
\hline
\end{tabular}

FS, focal sintomática; FPS, focal provavelmente sintomática; Gl, generalizada idiopática; GS, generalizada sintomática; GPS, generalizada provavelmente sintomática; P, ponta; PO, complexo de ponta-onda; PP, poliponta; AO, onda aguda; Gen, generalizada; Dif, difusa; HD, hemisfério direito; $D C T$, distúrbio cognitivo transitório; $A E$, atividade epileptiforme; CVAO, .dassificação visual de animais e objetos; MVMA, memória visual e memória auditiva; DC discriminação de cores; RP, reconhecimento de padrão; ET. Estimação de tempo. *menor proporção de respostas corretas durante a AE do que fora da $A E$ (qui-quadrado ou Fisher, $p \varangle 0,05$ ).

Tabela 3. Resultados do teste de pesquisa de distúrbio cognitivo transitório em 30 pacientes com atividade epileptiforme (AE) difusa ou generalizada

\begin{tabular}{lcc}
\hline Resultado & № de casos & $\%$ \\
\hline Com AE e com distúrbio cognitivo transitório & 13 & 43,3 \\
Com AE e sem distúrbio cognitivo transitório & 9 & 30,0 \\
Crise (ausência) durante o teste & 1 & 3,3 \\
Com AE rara & 7 & 23,3 \\
Total & 30 & \\
\hline
\end{tabular}

Em 3 pacientes não havia referência a crises epilépticas e os EEG foram realizados por queixa de cefaléia ou traumatismo crânio-encefálico.

Uma paciente (caso 23), com epilepsia mioclônica juvenil, havia sido submetida a dois EEG anteriores com testes cognitivos; em um deles apresentou mioclonias e, em outro, a AE era rara.

O tempo de aplicação dos testes, durante o EEG, variou de 9 a 63 minutos; as durações mais pro longadas corresponderam a pacientes com $A E$ rara, no sentido de tentar avaliar a ocorrência de DCT.

Tabela D/T/B consta a avaliação dos exames quanto à presença de distúrbio cognitivo transitório ou crise epiléptica. Nota-se que em 7 casos a AE foi rara (em geral menos que uma descarga em 5 minutos) e não permitiu avaliar a existência de DCT.

Em uma paciente (caso 30) ficou clinicamente evidente, para o observador, ter ocorrido uma au- sência durante o teste, sem que houvesse necessidade de correlação com o EEG.

O DCT foi caracterizado quando a AE ocorria: durante a apresentação do estímulo nos casos 9 e 27; na resposta, no caso 10, e tanto durante a apresentação quanto durante a resposta nos demais casos.

No caso 23, a Figura 1 mostra respostas incorretas em teste de discriminação de cores, durante descargas generalizadas de complexos de ponta-onda, com pequena duração (1 e 2 segundos). Neste caso também pôde ser observada resposta correta no início de uma descarga generalizada de 5 s de duração, logo seguida de erro no estímulo seguinte.

Na Figura 2 (caso 10), o DCT, em teste de memória verbal e auditiva, acompanha um complexo de onda aguda - onda lenta, difuso e isolado.

A morfologia do paroxismo mostrou influência na associação com DCT. Na Figura 3 (caso 28), a des- 


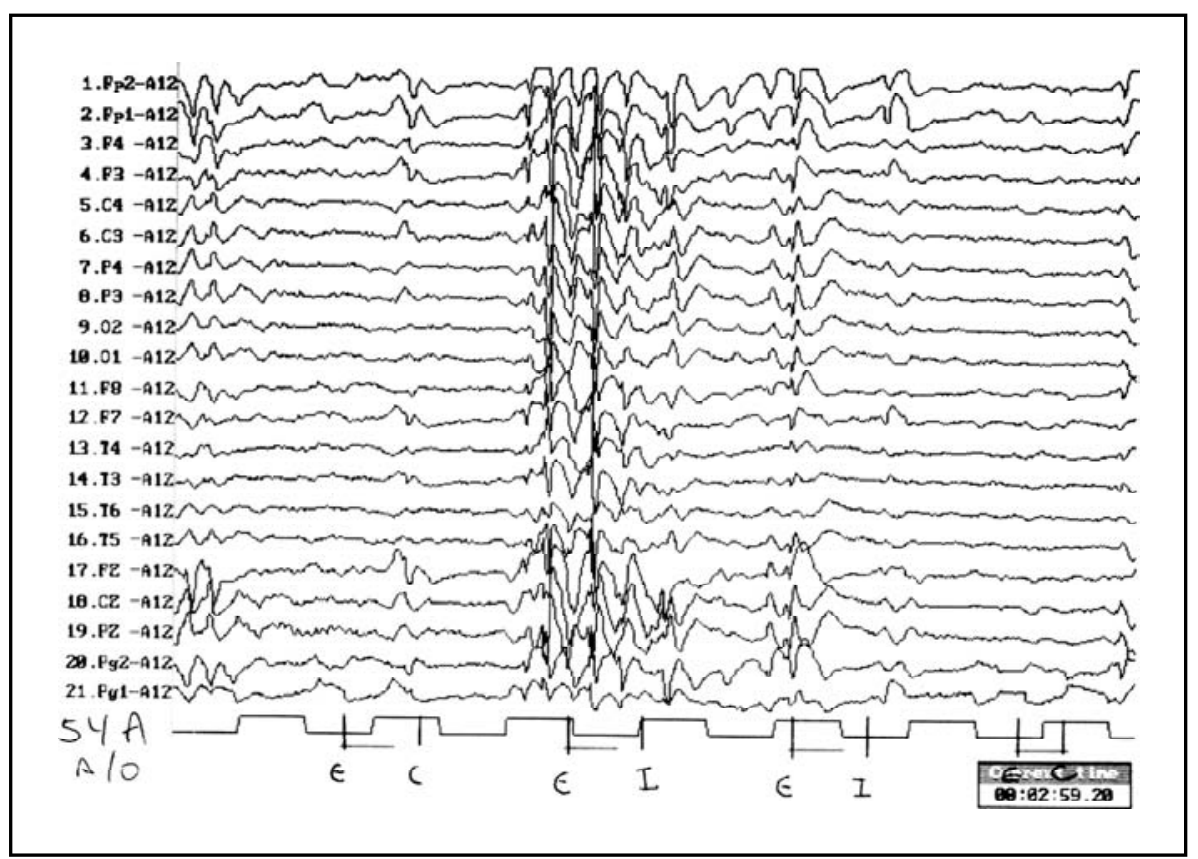

Fig 1. Respostas incorretas em teste de discriminação de cores durante descargas generalizadas de complexos de ponta-onda. Legenda: $E$, momento de apresentação do estímulo; $C$, momento da resposta correta; I, momento da resposta incorreta.

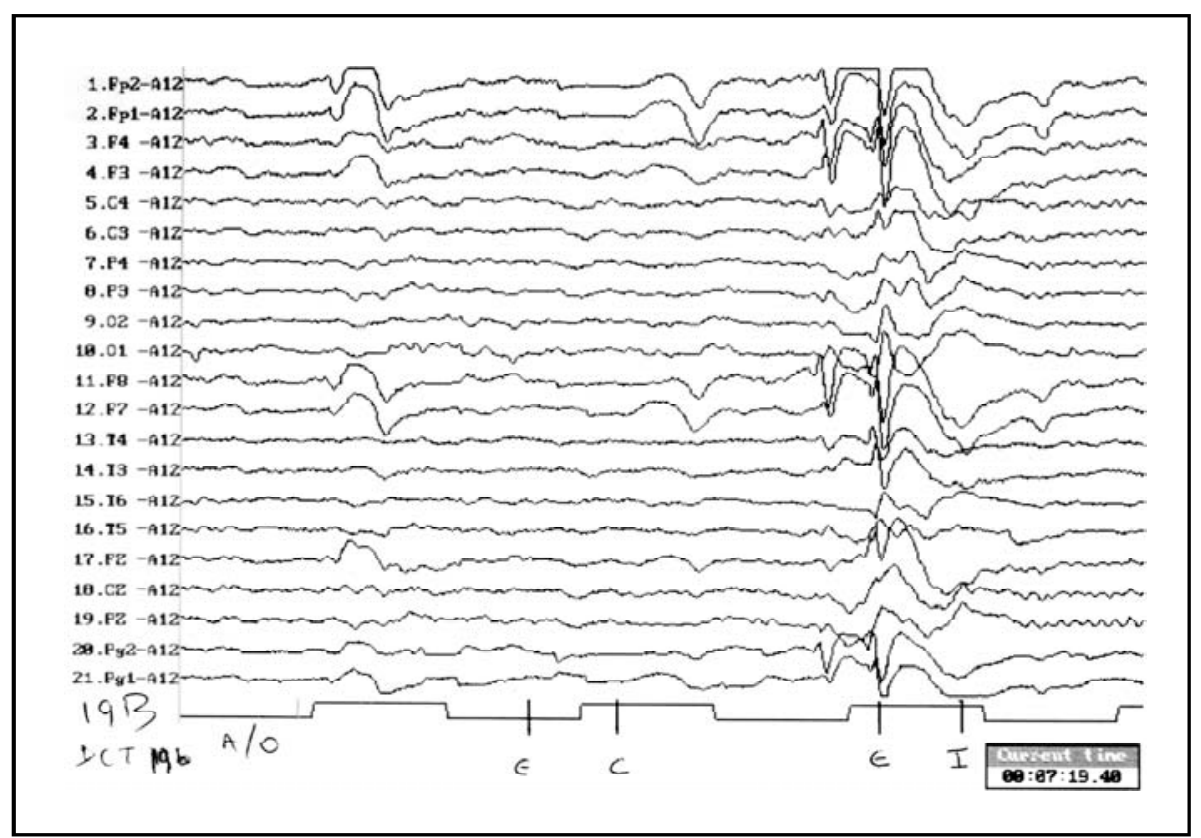

Fig 2. Resposta incorreta durante complexo de onda aguda-onda lenta difusa. Legenda: $E$, momento de apresentação do estímulo; $C$, momento da resposta correta; l, momento da resposta incorreta.

carga de ondas lentas com pontas de pequena amplitude associadas foi acompanhada de resposta in co reta, enquanto, logo a seguir, durante paroxismo de ondas lentas a reposta foi correta, em teste de reconhecimento de padrão.

Durante descargas generalizadas com duração de vários segundos, os erros são mais freqüentes, mas podem ocorrer, também, respostas corretas (caso 23).

Na evolução de uma descarga mais prolongada, sua modificação ao final já pode estar associada a acertos (caso 12).

DCT foi evidenciado mesmo durante um único complexo onda aguda - onda lenta difuso (caso 9). 


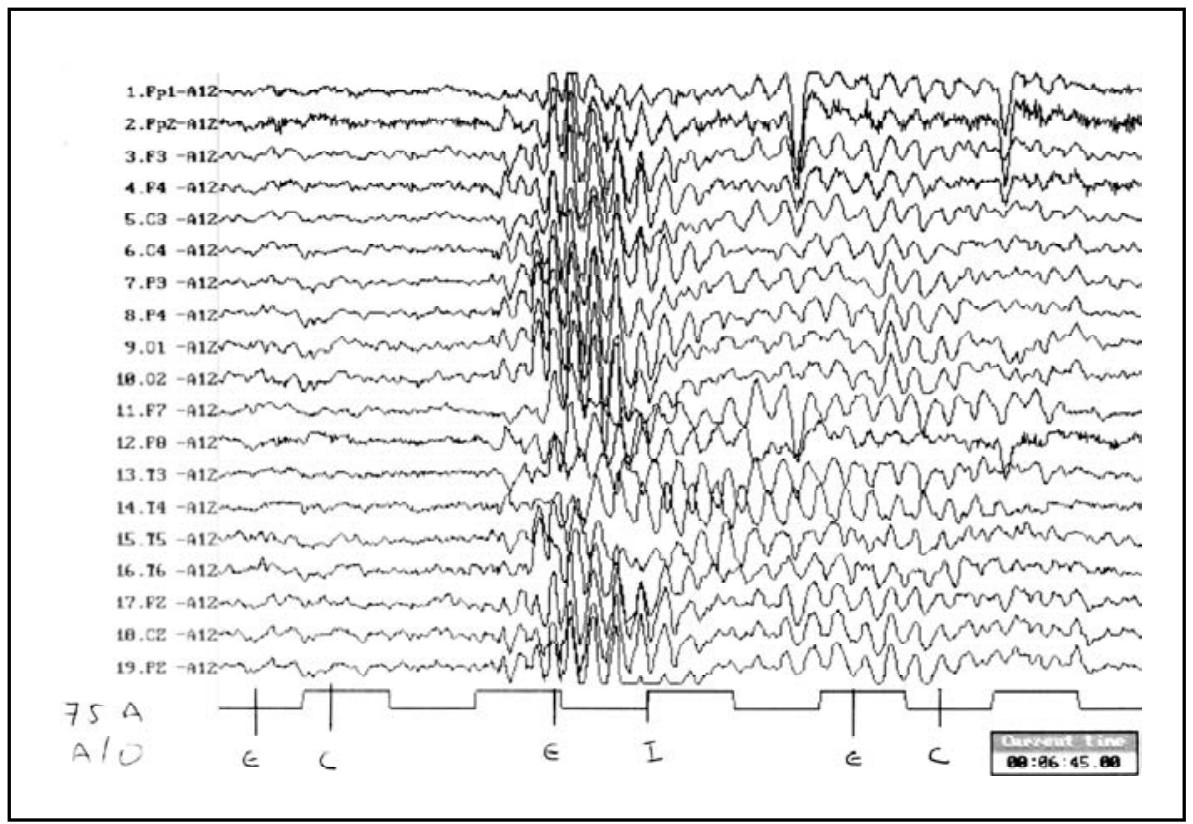

Fig 3. Resposta incorreta durante descarga de ondas lentas com pontas de pequena amplitude associadas e resposta correta em paroxismo de ondas lentas. Legenda: $E$, momento de apre sentação do estímulo; $C$, momento da resposta correta; l, momento da resposta incorreta.

Erros podem ser observados em descargas bilaterais relativamente localizadas e de curta duração (casos 5,13 e 27).

A extensão da descarga pode ser um fator imp o rtante na provocação de DCT. Assim, no caso 1, descargas anteriores e com predomínio lateralizado não eram acompanhadas por DCT, enquanto que aquelas com maior extensão já correspondiam a respostas incorretas, mesmo quando ocorriam somente no momento da emissão da resposta.

\section{DISCUSSÃO}

Apesar da inclusão na pesquisa de pacientes que tiveram EEG prévio com descargas relativamente numerosas, durante o EEG com as atividades cognitivas $23 \%$ dos pacientes tiveram descargas raras, o que impediu a avaliação da ocorrência ou não de DCT. Por vezes, essas descargas apareceram entre uma resposta e o estímulo seguinte, ou seja, em momento inadequado para a análise. Provavelmente houve inibição das descargas nas atividades cognitivas, fato também relatado em outras séries de pacientes com AE generalizada ${ }^{21,23}$.

É possível que a utilização, em cada paciente, de testes mais variadose de diferentes níveis de dificuldade, pudesse identificar os que ocasionassem menor redução de $A E^{25}$. Isto seria mais exeqüível se os exames fossem feitosem diferentes sessões, podendo assim estender o tempo de análise. Duração maior do perío do de análise, em sessões diferentes, p rovavelmente também possibilitaria o registro de maior número de descargase, conseqüentemente, maior probabilidade de identificação de DCT.

$\mathrm{O}$ desencadear de crises miodônicas, em um caso, está em acordo com a observação de Matsuoka et al. ${ }^{26}$, segundo a qual pacientes com crises miodônicas são vulneráveis a atividades mentais superiores requerendo ação, programação e pensamento. Noutro caso houve a caracterização clínica de uma ausência.

Nesta pesquisa os testes foram acompanhados por um dos autores já preparados para detecção de sinais de crises, mas não foi realizado vídeo-EEG, o que impediu a revisão posterior dosdados. É possível que algum episódio interpretado como DCT pudesse ser uma crise sutil, mas, nos momentos tidos como DCT, nem o paciente nem o pesquisador notaram sintomas/sinais de crise epiléptica.

Quanto ao tipo de prova, foram utilizadas semp reprovas contínuas, de acordo com as recomendações de Binnie et al. ${ }^{25}$. A prova de classificação visual de animais/objetos prestou-se adequadamente ao teste ao permitir grande número de estímulose res postas sem intervalos longos entre asfases. Já os tes tes de memória visual e auditiva, ao contarem com fase de repouso maior entre estímulos e respostas, foram mais longos e mais cansativos para o paciente. 
Observou-se, por vezes, maior número de descargas no período de repouso do que quando eram apresentados estímulos ou emitidas respostas.

Mesmo com essas dificuldades, foi possível caracterizar DCT em 43,3\% (13 de 30 casos) dos pacientes estudados e em $59,1 \%$ (13 de 22 casos) dos pacientes com AE suficiente para análise. Aarts et al. 7 , em estudo amplo e com metodologia rigorosa, observaram DCT em cerca de $50 \%$ dos pacientes.

A observação de diminuição no tempo de reação ou "não resposta" durante descargas generalizadas tem sido freqüentemente apontadas na literatura ${ }^{1,7}$, de modo mais constante quando a descarga dura mais de 3 segundos ${ }^{21}$. Foi possível em nosso estudo evidenciar DCT em vários casos com descargas de menos de $3 \mathrm{~s}$. Em um paciente (caso 10) resposta incorreta ocorreu durante complexos isolados de onda aguda-onda lenta. A ocorrência de respostas incorretas no início e no meio de desca rgas com vários segundos de duração (caso26) é referida por vários autores ${ }^{1,27}$.

Browne et al. ${ }^{1}$ obsenaram tempo de reação "anormal" durante a AE em $43 \%$ dos estímulos: para os estímulos apresentados nos primeiros $0,5 \mathrm{~s}$ da descarga, $20 \%$ eram anormais; a partir de $1,5 \mathrm{~s}$ havia algum acréscimo e estabilizavam-se em torno de $50 \%$ após 4 s. O comprometimento do tempo de reação era um pouco menor se o paroxismo não fosse totalmente generalizado. No primeiro segundo após a $A E$, o tempo de reação ainda era um pouco maior e só voltava ao normal 5 s apósa AE. Não observamos DCT no período imediatamente antes da descarga, embora isso tenha sido referido por Opp et al. ${ }^{27}$ em um de seus casos.

Em relação ao momento da descarga, vários au to resobservaram maior aumento de tempo de reação ou erros mais freqüentes quando a $\mathrm{AE}$ ocorria no período de reconhecimento do estímulo ${ }^{2-4,7}$, Na presente casuística, a descarga cobria o período de apresentação e de resposta na maioria dos casos, pois as provas foram montadas para tempos de reação pequenos. Em alguns casos, houve, no entanto, claro predomínio de DCT durante apresentação dos estímulose, em apenas um caso os erro s ocorreram em maior freqüência com descarga no momento da resposta. Para Hutt² a AE interferiria na memória operacional, havendo alguma degrada ção da percepção do estímulo no sistema nervoso.

Quanto à extensão da descarga, o caso 1 é esdarecedor, pois quando a AE era anterior e com pre- domínio unilateral, não havia DCT, e quando a área era mais extensa, já ocorriam respostas incorretas.

A morfologia do paroxismo mostrou influência na associação com DCT. No caso 28, por exemplo, os p a roxismos lentos estão associadosa respostas corretas e os paroxismos com pontas associadas correspondem a respostas incorretas. Tal achado está de acordo com Goode et al. ${ }^{21}$ que não observaram co $\mathrm{mp}$ rometimento de função durante paroxismos lentos, independentemente de sua duração.

O não achado de DCT em pacientes com descargas generalizadas ponta-onda também tem sido relatado por vários autore $\mathrm{s}^{1,21,23}$ e poderia decorrer do fato de áreas subcorticais reguladoras da atividade elétrica cortical e do nível de consciência puderam funcionar independentemente.

$O$ achado de DCT sugere que descargas elétricas cerebrais intercríticas, mesmo na ausência de crises, podem interferir nas atividades rotineiras dos pacientes como desempenho escolar e na direção de veículos $^{10-12}$.

Neste trabalho podemos observar que o DCT pode ser demonstrado numa parcela relativamente pequena dos pacientes com epilepsia, pois muitos foram exduídos por não apresentarem número suficiente de descargas no EEG. Foi possível evidenciar DCT em cerca de metade dos pacientes com AE freqüente em EEG anterior.

A ocorrência de DCT em pacientes sem epilepsia (dois dos casos deste trabalho) já foi assinalada

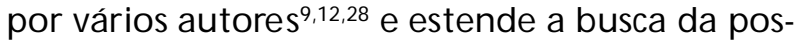
sível interferência de DCT com funções cognitivas para outras condições clínicas.

Uma questão de grande relevância é saber o quanto a AE pode ser responsável por comprometimento cognitivo de pacientes com epilepsia. Para avaliar a influência de vários fatores no estado cognitivo de pacientes, Aldenkamp e Arends ${ }^{13}$ estudaram um grupo particular com crises não convulsivas breves. Verificaram que o tipo de epilepsia tem impacto nas funções cognitivas estáveis como o desempenho educacional; já a AE e as crises (de modo mais intenso) comprometem processos cognitivos transitórios, em particular, alerta e vel o cidade mental. No entanto, os efeitos da AE e das crises podem ser cumulativos com o tempo e conseqüentemente afetar aspectos mais estáveis das funções cognitivas.

Assim o conceito de distúrbio cognitivo transitório é válido e deve haver preocupação de detec- 
ção precoce deste, assim como há necessidade de estudos longitudinais e prospectivos para tentar caracterizar em que situações clínicas a AE deve ser tratada independentemente das crises epilépticas ${ }^{18,19,29,30 .}$

\section{REFERÊNCIAS}

.1 B rowne TR. Penry JK, Porter RJ, Dreifuss FE. Responsiveness before, during and after spike-wave paroxysms. Neurology 1974;24:659-665.

2. Hutt SJ, Newton J, Fairweather H. Choice reaction time and EEG activity in children with epilepsy. Neuropsychologia 1977;15:257-267.

3. Hutt SJ, Fairweather H. Paced and unpaced serial response performance during two types of EEG activity. J Neurol Sci 1973;19:85-96.

4. Geller MR, Geller A. Brief amnestic effects of spike-wave discharges. Neurology 1970;20:380-381.

5. Siebelink BM, Bakker DJ, Binnie CD, Kasteleijn-Nolst Trenité DGA. Psychological effects of subclinical epileptiform EEG discharges in children: II. General intelligence tests. Epilespy Res 1988;2:117-121.

6. Guey J, Tassinari CA, Charles C, Coquery C. Variations du niveau d'efficience en relation avec des décharges épileptiques paroxystiques. Rev Neurol (Paris) 1965;112:311-317.

7. Aarts JHP, Binnie CD, Smit AM, Wilkins AJ. Selective cognitive impairment during focal and generalized epileptiform EEG activity. Brain 1984;107:293-308.

8. Shewmon DA, Erwin RJ. The effect of focal interictal spikes on perception and reaction time: II.Neuroanatomic specificity. Electroencephalogr Clin Neurophysiol 1988;69:338-352.

9. Kasteleijn-Nolst Trenité DGA, Siebelink BM, Berends SGC, van Strien JW, Meinardi H. Lateralized effects of subclincal epilpetiform EEG discharges on scholastic performance in children. Epilepsia 1990;31:740-746.

10. Stores G, Hart J. Reading skills of children with generalized or focal epilepsy attending ordinary school. Develop Med Child Neurol 1976; 18:705-716.

11. Kasteleijn-Nolst Trenité DGA, Bakker DJ, Binnie CD, Buerman A, Van Raaij M. Psychological effects of subclinical epileptiform EEG discharges on scholastic skills. Epilepsy Res 1988;2:111-116.

12. Kasteleijn-Nolst Trenité DGA, Riemersma JBJ, Binnie CD, Smit AM, Mei$n$ a rdi $H$. The influence of subclinical epileptiform EEG discharges on driving behaviour. Eletroencephalogr Clin Neurophysiol 1987;67:167-170.

13. Aldenkamp AP, Arends J. The relative influence of epileptic EEG discharges, short nonconvulsive seizures, and type of epilepsy on cognitive function. Epilepsia 2004;45:54-63.

14. Fonseca LC; Ted rus GMA. Pontas evocadas por estímulos somatossensitivos e atividade epileptiforme no eletrencefalograma em crianças normais. Arq Neuropsiquiatr 2003;61:793-795.
15. Harmony T, Becker J, Velasco M. Hallazgos electroencefalográficos en ninos "normales con bajo rendimiento escolar. A rch Invest Méd (Méx) 1987;18:229-307.

16. Holtmann M, Becker K, Kentner-Figura B, Schmidt MH. Increased frequency of rolandic spikes in ADHD children. Epilepsia 2003;44:1241-1244.

17. Dahlin M, Knutson E, Ämark P, Nergardh A. Reduction of epileptiform activity in response to low-dose clonazepam in children with epilepsy: a randomized double-blind study. Epilepsia 2000;41:308-315.

18. Engler F, Maeder-Ingvar M, Roulet E, Deonna T. Treatment with sulthiame (Ospolot) in benign partial epilepsy of childhood and related syndromes: an open clinical and EEG study. Neuropediatrics 2003; 34:105-109.

19. Pressler RM, Wilson AG, Robinson RO,Binnie CD. Treatment of interictal EEG discharges improves behaviour in children with epilepsy. Epilepsia 2002;43(Suppl 8):S44

20. Mendizabal JE, Nowack WJ. Transitory cognitive impairment with brief generalized spike-wavw paroxysm: a clinical counter example to the three-second rule. Clin Electroencephalog 1996;27:215-217.

21. Goode DJ, Penry JK, Dreifuss FE. Effects of paroxysmal spike-wave on continuous visual-motor performance. Epilepsia 1970;11:241-254.

22. Noachtar S, Sakamoto A, Westmorreland B. A glossary of terms most commonly used by clinical electroencephalographers and proposal for the report form for the EEG findings. Electroencephalogr Clin Neurofisiol 1999;52(Suppl):S21-S41.

23. Provinciali L, Signorino M, Censori B, Ceravolo G, Pesce MD. Recognition impairment correlated with short bisynchronous epileptic discharges. Epilepsia 1991;32,684-689.

24. Kasteleijn-Nolst Trenité DGA, Smit AM, Velis DN, Willemse, Boas WE On-line detection of transient neuropsychological disturbances during EEG discharges in children with epilepsy. Develop Med Child Neurol 1990;32:46-50.

25. Binnie CD, Channon S, Marston D. Learning disabilities in epilepsy: neurophysiological aspects. Epilepsia 1990;31(Suppl):S2-S8.

26. Matsuoka H, Takahashi T, Sasaki M, et al. Neuropsychological EEG activation in patients with epilepsy. Brain 2000;123:318-330.

27. Opp J, Wenzel D, Brandl U. Visuomotor coordination during focal and generalized EEG discharges. Epilepsia 1992;33:836-840.

28. Casas-Fernandez C, Belmonte-Avilés F, Fernández-Fernández, MV et al. Afectación cognitiva transitória por actividad electroencefalográfica paroxística subclinica. Rev Neurol 2002;35(Suppl 1):S21-S29.

29. Aldenkamp AP, Arends J. Effects of epileptiform EEG discharges on cognitive function: is the concept of "transient cognitive impairment" still valid? Epilepsy \& Behaviour 2003;5(Suppl 1):S25-S34

30. Binnie CD. Cognitive performance, subtle seizures, and the EEG. Epilepsia 2001;42(Suppl 1):S16-S18. 